\title{
A PROPERTY OF A SIMPLY ORDERED SET ${ }^{1}$
}

\author{
K. W. FOLLEY
}

Sierpinski ${ }^{2}$ has shown that the set of real numbers of the interval $(0,1)$ may be decomposed into $c$ disjoint subsets, each of power less than $c$, and such that the sum of every $c$ of these subsets has at least one point in common with every perfect subset of the interval.

The object of the present paper is to show that the same method of proof may be used to prove an analogous theorem concerning a more general type of set.

Definitions. $A$ simply ordered set $M$ is a set such that if any two of its elements are given it is known which one precedes.

$A$ subset of $M$ is said to be cofinal (coinitial) with $M$ if no element of $M$ follows (precedes) all the elements of the subset.

$A n \eta_{\alpha}$ subset of $M$ is one which is neither cofinal nor coinitial with any subset of $M$ of power less than $\boldsymbol{\aleph}_{\alpha}$ and which contains no pair of neighboring subsets both of which have power less than $\boldsymbol{\aleph}_{\alpha}$.

Various properties of simply ordered sets $M$ containing everywhere dense $\eta_{\alpha}$ subsets, including a discussion of the perfect subsets of $M$, were discussed by the writer in a previous paper. ${ }^{3}$

THEOREM 1. Let $M$ be a simply ordered set containing an everywhere dense $\eta_{\alpha}$ subset $N$. There exists a decomposition of $M$ into $2^{\mathbf{N}_{\alpha}}$ disjoint subsets, each of power less than $2 \mathbf{\aleph}_{\alpha}$, and such that the sum of every $2 \mathbf{N}_{\alpha}$ of these subsets has at least one point in common with every perfect subset of $M$.

Proof. Let $\phi$ be the smallest ordinal number of power $2 \aleph_{\alpha}$. A transfinite sequence of type $\phi$ formed of all the points of $M$ exists, namely,

$$
m_{1}, m_{2}, m_{3}, \cdots, m_{\xi}, \cdots, \quad \xi<\phi .
$$

The perfect subsets of $M$ having power $2 \aleph_{\alpha}$ may be arranged in the form of a transfinite sequence of type $\phi$ as follows:

$$
M_{1}, M_{2}, M_{3}, \cdots, M_{\xi}, \cdots, \quad \xi<\phi .
$$

Let us now define a transfinite sequence $\left\{H_{\xi}\right\}_{\xi<\phi}$ of subsets of $M$ : $H_{1}$ is formed of the single element $m_{1}$.

\footnotetext{
1 Presented to the Society, November 22, 1940.

${ }^{2}$ Fundamenta Mathematicae, vol. 24 (1935), pp. 8-11.

3 Proceedings of the Royal Society of Canada, Section III, vol. 22 (1928), pp. 225-239.
} 
Now, let $\beta$ be any ordinal number between 1 and $\phi$. Suppose we have defined all sets $H_{\xi}$, where $\xi<\beta$, and suppose further that the power of $H_{\xi}$ is not greater than $\bar{\xi}$. Let $S_{\beta}=\sum_{\xi<\beta} H_{\xi}$. Then $S_{\beta}$ is evidently of power not greater than $\bar{\beta}^{2}<2 \aleph_{\alpha}$.

We shall define a transfinite sequence $\left\{p_{\xi}^{\beta}\right\}$ as follows. $M_{1}$ being of power $2^{\aleph_{\alpha}}$, and $S_{\beta}$ being of power less than $2^{\aleph_{\alpha}}, M_{1}-S_{\beta}$ contains points. Let $p_{1}^{\beta}$ be the first element of (1) which belongs to $M_{1}-S_{\beta}$. Now let $\eta$ be any ordinal number between 1 and $\beta$, and let us suppose we have already defined the points $p_{\xi}^{\beta}$, where $\xi<\eta$. Let all these points form the set $T_{\eta}^{\beta}$ of power not greater than $\bar{\eta} \leqq \bar{\beta}<2^{\aleph_{\alpha}}$. The set $M_{\eta}-\left(S_{\beta}+T_{\eta}\right)$ contains points. Let $p_{\eta}^{\beta}$ be the first point of (1) which belongs to the set $E_{\eta}-\left(S_{\beta}+T_{\eta}\right)$.

Define $H_{\beta}$ as the set of all points $p_{\xi}^{\beta}$, where $\xi<\beta$; it is a set of power not greater than $\bar{\beta}<2 \aleph_{\alpha}$.

The sets $H_{\xi}$, with $\xi<\phi$, are thus defined by transfinite induction. They are evidently disjoint subsets of $M$, each of power less than $2^{\boldsymbol{N}_{\alpha}}$. The set $R=M-\sum_{\xi<\phi} H_{\xi}$ is of power not greater than $2 \boldsymbol{s}_{\alpha}$. Hence the elements of $R$ may be arranged in the form of a transfinite sequence of type $\psi \leqq \phi$ as follows:

$$
q_{1}, q_{2}, q_{3}, \cdots, q_{\xi}, \cdots, \quad \xi<\psi .
$$

Let $N_{\xi}=H_{\xi}+q_{\xi}$ for $\xi<\psi$, and if $\psi<\phi, M_{\xi}=H_{\xi}$ for $\psi \leqq \xi<\phi$.

The sets $N_{\xi}$ are disjoint subsets of $M$, each of power less than $2 \aleph_{\alpha}$. Moreover $M=\sum_{\xi<\phi} N_{\xi}$.

Now, let $F$ be the sum of any $2 \boldsymbol{N}_{\alpha}$ of the sets $N_{\xi}, \xi<\phi$. If $\mu$ is any ordinal number less than $\phi$, there is an ordinal number $\beta$ such that $\mu<\beta<\phi$, and such that $N_{\beta}$ belongs to $F$. Since $\beta>\mu$ we have $p_{\mu}^{\beta}$ belonging to $H_{\mu}$ and therefore to $N_{\mu}$. Besides $p_{\mu}^{\beta}$ belongs to $M_{\mu}$. Hence $p_{\mu}^{\beta}$ is a point of $M_{\mu} \cdot H_{\beta}$ and so of $F \cdot M_{\mu}$.

It follows that $F$ has at least one point in common with every subset $M_{\xi}$ of (2), which was to be proved.

THEOREM 2. The generalized hypothesis of the continuum $\left(2 \boldsymbol{\aleph}_{\alpha}=\boldsymbol{N}_{\alpha+1}\right)$ is equivalent to the following statement:

The set $M$ may be decomposed into disjoint subsets, each of power not greater than $\boldsymbol{\aleph}_{\alpha}$, such that the sum of any class of more than $\boldsymbol{\aleph}_{\alpha}$ of them has at least one point in common with every perfect subset of $M$.

Proof. (a) If the generalized hypothesis of the continuum is assumed, Theorem 1 becomes the second part of Theorem 2 .

(b) Suppose that the second part of Theorem 2 is true. There are $2^{\aleph_{\alpha}}$ perfect subsets of ${ }^{4} M$. Each disjoint subset of $M$ having a power

${ }^{4}$ See K. W. Folley, loc. cit., p. 232. 
not greater than $\boldsymbol{\aleph}_{\alpha}$, it follows that a class of $\boldsymbol{\aleph}_{\alpha+1}$ of them has power $\boldsymbol{\aleph}_{\alpha+1}$. Such a class has at least one point in common with every perfect subset of $M$. Thus, there results the inequality

$$
\aleph_{\alpha+1} \leqq 2^{\aleph_{\alpha}} \text {. }
$$

The inequality in the opposite sense being well known, the generalized hypothesis of the continuum follows.

WAYNE UNIVERSITY

\section{UNIQUENESS THEOREMS FOR RATIONAL FUNCTIONS ${ }^{1}$}

\section{FRITZ HERZOG}

In his book on the theory of meromorphic functions, ${ }^{2} \mathrm{R}$. Nevanlinna proved a number of "uniqueness theorems." The most important of them ${ }^{3}$ states that if two functions $w=f(x)$ and $w=g(x)$, meromorphic in the whole $x$-plane, assume five values of $w$ (finite or infinite) at the same points $x$ they must be identical. If we understand by the distribution of a function $w=\phi(x)$ with respect to a given value of $w$ simply the set of all points $x$ where $\phi(x)$ assumes that value $w$, regardless of multiplicity, we may state the above theorem in the following way: Two meromorphic functions which have identical distributions with respect to five values of the dependent variable must be identical. In proving this theorem, Nevanlinna explicitly assumes the functions to be transcendental (i.e., not rational). ${ }^{3}$ It is trivial, however, that the theorem would apply to two rational functions $w=f(x)$ and $w=g(x)$ as well, which can be easily seen by considering the transcendental functions $w=f\left(e^{x}\right)$ and $w=g\left(e^{x}\right)$.

The example of the functions $w=e^{x}$ and $w=e^{-x}$, which have identical distributions with respect to the four values $w=1,-1,0, \infty$, shows that five is the smallest number for which the above-mentioned uniqueness theorem holds true. It will be shown in this paper that such is not the case for rational functions for which five may, indeed, be replaced by four. (See Theorem I.)

The question arises as to what can be said about two rational functions that have identical distributions with respect to only three

\footnotetext{
1 Presented to the Society, February 24, 1940.

${ }^{2}$ Rolf Nevanlinna, Le Théorème de Picard-Borel et la Thêrie des Fonctions Méromorphes, Paris, 1929.

${ }^{3}$ See loc. cit., p. 109.
} 